\title{
Pré-natal na atenção primária, adequação das consultas e avaliação da assistência às gestantes: revisão integrativa
}

RESUMO | Objetivo: avaliar a assistência às gestantes na atenção primária à saúde e a adequação das consultas de pré-natal. Método: trata-se de uma revisão integrativa de literatura, descritiva de abordagem qualitativa, realizada no ano de 2019. Utilizouse artigos da Biblioteca Virtual em Saúde (BVS), através dos seguintes descritores padronizados no DeCS: qualidade da assistência à saúde, cuidado pré-natal e saúde da mulher, resultando em 107 artigos. Após filtragem com os critérios de inclusão/exclusão, seguida de uma leitura criteriosa em resposta ao problema de pesquisa, totalizou numa amostra de 14 artigos. Resultados: destes, onze artigos tiveram em suas conclusões a necessidade de mudanças no acompanhamento pré-natal. Conclusão: a pesquisa possibilitou a visualização das dificuldades relacionadas à gestão, a união da equipe multiprofissional, da capacitação e aperfeiçoamento dos profissionais de saúde, da melhoria das consultas de pré-natal e também da diferença na qualidade da assistência prestada às mulheres negras e indígenas.

Palavras-chaves: Cuidado pré-natal; Qualidade da assistência à saúde; Saúde da mulher.

\begin{abstract}
Objective: to evaluate the assistance to pregnant women in primary health care and the adequacy of prenatal consultations. Method: this is an integrative literature review, descriptive of a qualitative approach, carried out in 2019. Articles from the Virtual Health Library (VHL) were used, using the following standardized descriptors in DeCS: quality of health care, prenatal care and women's health, resulting in 107 articles. After filtering with the inclusion / exclusion criteria, followed by a careful reading in response to the research problem, it totaled a sample of 14 articles. Results: of these, eleven articles had in their conclusions the need for changes in prenatal care. Conclusion: the research made it possible to visualize the difficulties related to management, the unification of the multidisciplinary team, the training and improvement of health professionals, the improvement of prenatal consultations and also the difference in the quality of care provided to black and indigenous women.
\end{abstract}

Keywords: Prenatal care; Quality of health care; Women's health.

RESUMEN | Objetivo: evaluar la atención a la gestante en la atención primaria de salud y la adecuación de las consultas prenatales. Método: se trata de una revisión de literatura integradora, descriptiva de abordaje cualitativo, realizada en 2019. Se utilizaron artículos de la Biblioteca Virtual en Salud (BVS), utilizando los siguientes descriptores estandarizados en DeCS: calidad de la atención en salud, atención prenatal y salud de la mujer, resultando en 107 artículos. Luego de filtrar con los criterios de inclusión / exclusión, seguido de una lectura atenta en respuesta al problema de investigación, totalizó una muestra de 14 artículos. Resultados: de estos, once artículos tenían en sus conclusiones la necesidad de cambios en la atención prenatal. Conclusión: la investigación permitió visualizar las dificultades relacionadas con la gestión, la unificación del equipo multidisciplinario, la formación y perfeccionamiento de los profesionales de la salud, el mejoramiento de las consultas prenatales y también la diferencia en la calidad de la atención brindada a mujeres negras e indígenas.

Palabras claves: Atención prenatal; Calidad de laatención médica; Lasalud de lamujer.

\section{Adriene de Freitas Moreno Rodrigues}

Enfermeira, Mestra em Gestão Integrada do Território pela Universidade Vale do Rio Doce - MG, Professora dos cursos de saúde do Centro Universitário do Espírito Santo - UNESC. Colatina - ES, Brasil. Pesquisadora do Grupo de Pesquisa Território Saúde e Sociedade.

ORCID: 0000-0001-5028-3262

\section{Cecília Lievore Candido}

Enfermeira - Centro Universitário do Espírito Santo - UNESC. Enfermeira da Clínica Cirúrgica do Hospital e Maternidade São José, Colatina - ES, Brasil.

ORCID ID: 0000-0003-3401-4067

\section{Greice Kelly Palmeira Campos}

Acadêmica de Medicina - Centro Universitário do Espírito Santo - UNESC, Colatina-ES, Brasil.

ORCID ID: 0000-0002-5169-5282

\section{José Emílio Simoura Barcellos}

Enfermeiro - Centro Universitário do Espírito Santo - UNESC. Enfermeiro da ClínicaMédica do Hospital São Bernardo Saúde, Colatina - ES, Brasil.

ORCID ID: 0000-0002-1865-9300

Recebido em: 19/07/2020

Aprovado em: 25/01/2021

\section{Luciano Antonio Rodrigues}

Enfermeiro, Doutor em Ciências da Saúde, Universidade do Extremo Sul Catarinense. Professor dos cursos de saúde do Centro Universitário do Espírito Santo UNESC. Colatina - ES, Brasil. Pesquisadoro Líder do Grupo de Pesquisa Território Saúde e Sociedade.

ORCID ID: 0000-0001-5758-456X

\section{Tássia Sabrina Seidel}

Acadêmica de Enfermagem Iniciação Científica - Centro Universitário do Espírito Santo Colatina - ES, Brasil.

ORCID ID: 0000-0002-3276-3542 
INTRODUÇÃO

A qualidade da assistência à saúde de gestantes necessita mais do que a solução de problemas ou a obtenção de recursos tecnológicos. Para garantir uma atenção humanizada, devem-se seguir princípios como o acesso das usuárias aos três níveis de assistência, inclusão de sistemas de referência e contrareferência, capacitação dos profissionais e disposição dos recursos tecnológicos ${ }^{1,2,3,4}$.

No intuito de melhorar a assistência prestada à saúde da mulher no âmbito público, o Ministério da Saúde em 1983, instituiu o Programa de Assistência Integral à Saúde da Mulher (PAISM), através do movimento de mulheres juntamente com os profissionais da área da saúde, assegurando às mulheres o direito por lei à saúde $\mathrm{e}^{5,6,7,8,9,10}$.

Em 1991, foi elaborado a "Política Nacional de Atenção Integral à Saúde de Mulher - Princípios e Diretrizes", que propõe medidas de humanização e qualidade do atendimento às mulheres ${ }^{1}$. Posteriormente, o Ministério da Saúde, em $1^{\circ}$ de junho de 2000, institui o "Programa de Humanização no Pré-natal e Nascimento" (PHPN) ${ }^{11,12}$. O qual veio para estruturar a atenção dada às mulheres durante as consultas de pré-natal.

Em setembro de 2000, o Brasil se dispôs a cumprir os "Objetivos de Desenvolvimento do Milênio" propostos pela ONU, o qual possuía como um dos objetivos a redução da taxa de mortalidade materna. Entretanto, a ONU relatou que o Brasil teve sua nota abaixo da meta devido à baixa fidedignidade com as estatísticas de saúde nacionais ${ }^{4}$. Com a finalidade de diminuir essa taxa, foi criada a Rede Cegonha em 2011, assegurando à mulher o direito ao planejamento reprodutivo e à atenção humanizada à gravidez, ao parto e ao puerpério ${ }^{11,13}$.

As mortes maternas e/ou fetais durante o parto podem, na maioria das vezes, ser evitadas se o pré-natal for bem realizado. Uma assistência de qualidade, com

boa estrutura e atendimento holístico, gera bons resultados ${ }^{14,15,16}$. Assim, a avaliação ampla das consultas de pré-natal realizadas nas unidades básicas analisando esses quesitos, irá conceder às gestantes, aos profissionais e ao governo um progresso $^{1,13,17}$.

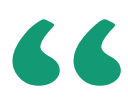

A qualidade da assistência à saúde de gestantes necessita mais do que a soluçãa
de problemas
ou a obtenção
de recursos
tecnológicos.

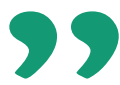

Em 2015, a ONU anunciou "17 Objetivos de Desenvolvimento Sustentável" que visam uma melhor qualidade de vida para todos. Entre eles, está incluso o objetivo de número 3: "Assegurar uma vida saudável e promover o bem-estar para todos, em todas as idades", que dispõe sobre saúde materna, infantil e doenças transmitidas verticalmente. Dessa maneira, pretende-se reduzir as taxas de mortalidade materna para menos de 70 mortes por 100.000 nascidos vivos até 203018 .

Nas últimas décadas, houve uma diminuição dos índices de mortalidade materna, contudo ainda é preocupante esta realidade social no país ${ }^{16}$. Assim, justifica-se a importância deste estudo para as mulheres e futuras gestantes, para os profissionais de saúde e para o Estado, a fim de identificar os possíveis erros na atenção à saúde das mulheres durante o período gravídico e incentivar a melhoria da assistência.

\section{MÉTODO}

Trata-se de uma revisão integrativa de literatura, descritiva de abordagem qualitativa, realizada no ano de 2019. Para a elaboração deste estudo seguiu-se as 6 etapas da revisão integrativa: Identificação do tema, seleção da questão da pesquisa, estabelecimento dos critérios de inclusão e exclusão, categorização dos estudos selecionados, análise e interpretação dos resultados e apresentação da revisão-síntese de conhecimento.

A questão norteadora para a composição da pesquisa foi: As consultas de pré-natal e a assistência às gestantes na atenção primária à saúde são adequadas?

Para compor a amostra foram utilizados os artigos encontrados na Biblioteca Virtual em Saúde (BVS) do banco de dados da Medline, LILACS, BDENF e BBO. Para maior efetividade da análise foram utilizados descritores padronizados no DeCS (Descritores da Ciência da Saúde): Qualidade da Assistência à Saúde, Cuidado Pré-natal e Saúde da Mulher, agrupados em trio ao operador boleano AND.

Os critérios de inclusão no estudo incluíam artigos na íntegra na base de dados supracitada, disponíveis gratuitamente, no idioma português, publicados no Brasil e no período compreendido entre 2015 a 2019. Como critérios de exclusão, artigos que não respondessem a questão norteadora do estudo, artigos duplicados e que estivessem fora da base de dados pesquisada

Na primeira etapa, utilizando os descritores, foram encontrados no banco de dados da BVS, 107 artigos. Após adicionar os filtros com os critérios de inclusão, a pesquisa resultou em 35 artigos. Estes, 
após leitura criteriosa se resumiram a uma amostra de 14 artigos. Os resultados encontrados na pesquisa foram organizados e apresentados em forma de quadro contendo as seguintes variáveis: título do artigo, autores/ ano de publicação, periódico, objetivos e principais conclusões (quadro 1). Os dados foram validados por dois pesquisadores. Como endosso de todos os itens da revisão foi utilizado a pro-

\section{Figura 1. Fluxograma e procedimentos utilizados para seleção dos artigos. Colatina,} ES, Brasil, 2019.

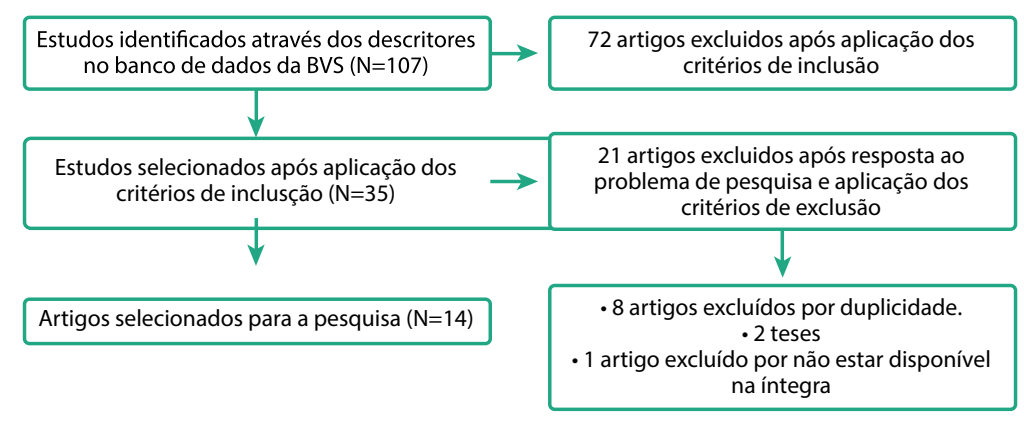

tocolo PRISMA de 27 itens de avaliação.

O estudo foi fomentado pelos próprios pesquisadores não havendo financiamento por agência externa de patrocínio de pesquisa.

\section{RESULTADOS}

Após classificação de todo o material, os dados foram organizados, facilitando a visualização e compreensão do processo seletivo do material como apresenta a figura 1.

O Quadro 1 representa as especificações de cada um dos artigos selecionados. Está descrito o título de cada artigo, os nomes dos autores e ano de publicação, o periódico que o artigo foi publicado, o objetivo, o método e/ou tipo de pesquisa que foi realizado para a construção do artigo e as principais conclusões.

Quadro 1. Descrição das variáveis dos artigos selecionados (N=14). Colatina, ES, Brasil, 2019.

\begin{tabular}{|c|c|c|c|c|}
\hline TÍTULO DO ARTIGO & $\begin{array}{l}\text { AUTOR/ } \\
\text { ANO }\end{array}$ & PERIÓDICO & OBJETIVOS & PRINCIPAIS CONCLUSÕES \\
\hline $\begin{array}{l}\text { A qualidade de } \\
\text { uma rede integrada: } \\
\text { acessibilidade e co- } \\
\text { bertura no pré-natal }\end{array}$ & $\begin{array}{l}\text { Silva } \\
\text { et al. }{ }^{6} \\
(2015)\end{array}$ & $\begin{array}{l}\text { Rev. Pesqui. } \\
\text { Cuid. Fundam. }\end{array}$ & $\begin{array}{l}\text { Analisar os valores expressos } \\
\text { no discurso das gestantes sob } \\
\text { a acessibilidade dos exames do } \\
\text { acompanhamento do pré-natal. }\end{array}$ & $\begin{array}{l}\text { Considera-se, como primordial, que se estabeleça essa } \\
\text { meta para a humanização do cuidado, cabendo adotar } \\
\text { estratégias de participação popular, além da gestão mu- } \\
\text { nicipal propiciar as condições necessárias como espaço } \\
\text { físico e equipamentos. }\end{array}$ \\
\hline $\begin{array}{l}\text { Assistência ao pré- } \\
\text {-natal e as práticas } \\
\text { desenvolvidas pela } \\
\text { equipe de saúde: } \\
\text { revisão integrativa }\end{array}$ & $\begin{array}{l}\text { Santia- } \\
\text { goet al. }{ }^{8} \\
(2017)\end{array}$ & $\begin{array}{l}\text { Rev. Pesqui. } \\
\text { Cuid. Fundam. }\end{array}$ & $\begin{array}{l}\text { Apreender as práticas comumente } \\
\text { desenvolvidas na estratégia saúde } \\
\text { da família durante o pré-natal. }\end{array}$ & $\begin{array}{l}\text { Os profissionais de saúde devem trabalhar na perspecti- } \\
\text { va da multidisciplinaridade, na qual o cuidado aconteça } \\
\text { de forma integral e complementar. }\end{array}$ \\
\hline $\begin{array}{l}\text { Avaliação da } \\
\text { assistência com foco } \\
\text { na consulta de aten- } \\
\text { dimento pré-natal }\end{array}$ & $\begin{array}{l}\text { Ferreira } \\
\text { et al. }{ }^{9} \\
(2017)\end{array}$ & Rev. Ciênc. Plur. & $\begin{array}{l}\text { Avaliar a qualidade da assistência } \\
\text { prestada às gestantes nas consul- } \\
\text { tas de atendimento pré-natal na } \\
\text { cidade de Santa Cruz/RN, Brasil. }\end{array}$ & $\begin{array}{l}\text { A assistência pré-natal tem sido realizada de forma } \\
\text { positiva, tendo em vista o cumprimento de condutas } \\
\text { preconizadas pelos programas de saúde da mulher. }\end{array}$ \\
\hline $\begin{array}{l}\text { Avaliação dos indi- } \\
\text { cadores de processo } \\
\text { do Programa de } \\
\text { Humanização no } \\
\text { Pré-Natal e Nasci- } \\
\text { mento e da Rede } \\
\text { Cegonha }\end{array}$ & $\begin{array}{l}\text { Maia } \\
\text { et al. }{ }^{10} \text {. } \\
(2017)\end{array}$ & $\begin{array}{l}\text { Rev. Pesqui. } \\
\text { Cuid. Fundam. }\end{array}$ & $\begin{array}{l}\text { Analisar os indicadores de processo } \\
\text { do Programa de Humanização no } \\
\text { Pré-Natal e Nascimento (PHPN) e } \\
\text { da Rede Cegonha a partir do siste- } \\
\text { ma de informação de pré-natal. }\end{array}$ & $\begin{array}{l}\text { São necessárias intervenções constantes pela gestão } \\
\text { para melhoria da qualidade da assistência pré-natal. }\end{array}$ \\
\hline
\end{tabular}




\begin{tabular}{|c|c|c|c|c|}
\hline $\begin{array}{l}\text { Avaliação da Assis- } \\
\text { tência pré-natal em } \\
\text { município do Sul do } \\
\text { Brasil }\end{array}$ & $\begin{array}{l}\text { Segatto } \\
\text { et al. }{ }^{11} \\
(2015)\end{array}$ & $\begin{array}{l}\text { Rev. Enferm. } \\
\text { UFPI. }\end{array}$ & $\begin{array}{l}\text { Verificar a efetividade da assistên- } \\
\text { cia pré-natal por meio de indicado- } \\
\text { res de processo de um município } \\
\text { da região Sul do Brasil. }\end{array}$ & $\begin{array}{l}\text { Os resultados sinalizam para uma assistência incomple- } \\
\text { ta no pré-natal, que pode estar associada à subnotifica- } \\
\text { ção do acompanhamento. }\end{array}$ \\
\hline $\begin{array}{l}\text { Avaliação da aten- } \\
\text { ção ao pré-natal na } \\
\text { atenção básica no } \\
\text { Brasil }\end{array}$ & $\begin{array}{l}\text { Cunha } \\
\text { et al.1.12 } \\
\text { (2019) }\end{array}$ & $\begin{array}{l}\text { Rev. Bras. } \\
\text { Saúde Mater. } \\
\text { Infant. }\end{array}$ & $\begin{array}{l}\text { Avaliar a atenção ao pré-natal na } \\
\text { Atenção Básica identificando os } \\
\text { aspectos que influenciam a ade- } \\
\text { quação estrutural e operacional. }\end{array}$ & $\begin{array}{l}\text { Uma adequada atenção ao pré-natal precisa ser inte- } \\
\text { gral com fortalecimento das redes regionais voltadas } \\
\text { para a inclusão social. }\end{array}$ \\
\hline $\begin{array}{l}\text { Avaliação da } \\
\text { atenção pré-natal } \\
\text { ofertada às mulhe- } \\
\text { res indígenas no } \\
\text { Brasil: achados do } \\
\text { Primeiro Inquérito } \\
\text { Nacional de Saúde e } \\
\text { Nutrição dos Povos } \\
\text { indígenas }\end{array}$ & $\begin{array}{l}\text { Garnelo } \\
\text { et al. }{ }^{13} \\
(2019)\end{array}$ & $\begin{array}{l}\text { Cad. Saúde } \\
\text { Pública. }\end{array}$ & $\begin{array}{l}\text { Analisa os dados coletados no } \\
\text { âmbito do Inquérito Nacional no } \\
\text { Brasil com vistas a avaliar, por } \\
\text { meio de indicadores selecionados, } \\
\text { a atenção pré-natal oferecida às } \\
\text { mulheres indígenas no país. }\end{array}$ & $\begin{array}{l}\text { Os resultados reafirmam a persistência de desigual- } \\
\text { dades étnico-raciais que comprometem a saúde e o } \\
\text { bem-estar de mães indígenas. }\end{array}$ \\
\hline $\begin{array}{l}\text { Desigualdades } \\
\text { sócio-demográficas } \\
\text { e na assistência } \\
\text { à maternidade } \\
\text { entre puérperas no } \\
\text { Sudeste do Brasil } \\
\text { segundo cor da pele: } \\
\text { dados do Inquérito } \\
\text { Nacional Nascer no } \\
\text { Brasil (2011-2012) }\end{array}$ & $\begin{array}{l}\text { Diniz } \\
\text { et }^{1} .^{14} \\
(2016)\end{array}$ & Saúde Soc. & $\begin{array}{l}\text { Analisar as mudanças nas desi- } \\
\text { gualdades sociodemográficas e } \\
\text { na assistência à maternidade no } \\
\text { Sudeste do Brasil, segundo raçal } \\
\text { cor, na última década. }\end{array}$ & $\begin{array}{l}\text { Ainda que importantes disparidades persistam, houve } \\
\text { alguma redução das diferenças sociodemográficas e um } \\
\text { aumento do acesso, tanto a intervenções adequadas } \\
\text { quanto às desnecessárias e potencialmente danosas. }\end{array}$ \\
\hline $\begin{array}{l}\text { Processo e resultado } \\
\text { do cuidado pré-natal } \\
\text { segundo os modelos } \\
\text { de atenção primária: } \\
\text { estudo de coorte }\end{array}$ & $\begin{array}{l}\text { Oliveira, } \\
\text { Ferrari e } \\
\text { Parada' } \\
(2019)\end{array}$ & $\begin{array}{l}\text { Rev. Lat. Am. } \\
\text { Enfermagem }\end{array}$ & $\begin{array}{c}\text { Avaliar indicadores de processo } \\
\text { e resultado do cuidado pré-natal } \\
\text { desenvolvido na atenção primária } \\
\text { comparando os modelos de aten- } \\
\text { ção tradicional e Estratégia Saúde } \\
\text { da Família. }\end{array}$ & $\begin{array}{l}\text { Possivelmente, a melhor qualidade da atenção pré-natal } \\
\text { foi capaz de minimizar efeitos socioeconômicos } \\
\text { negativos. }\end{array}$ \\
\hline $\begin{array}{l}\text { Programa mais } \\
\text { médicos: qualifica- } \\
\text { ção da atenção ao } \\
\text { pré-natal e puer- } \\
\text { pério no âmbito da } \\
\text { estratégia de saúde } \\
\text { da família }\end{array}$ & $\begin{array}{l}\text { Vidal, } \\
\text { Santos e } \\
\text { Prado }^{16} \\
(2017)\end{array}$ & Rev. APS & $\begin{array}{l}\text { Melhorar a atenção ao pré-natal e } \\
\text { puerpério na área de abrangência } \\
\text { da equipe. }\end{array}$ & $\begin{array}{l}\text { Os profissionais ficaram mais capacitados para atender } \\
\text { as gestantes e puérperas com conhecimentos atuali- } \\
\text { zados e sensibilização sobre a atuaçãa no trabalho da } \\
\text { atenção primária. }\end{array}$ \\
\hline $\begin{array}{l}\text { Representações so- } \\
\text { ciais sobre o acesso } \\
\text { e o cuidado pré-na- } \\
\text { tal no Sistema Único } \\
\text { de Saúde da Região } \\
\text { Metropolitana da } \\
\text { Grande Vitória, } \\
\text { Espírito Santo }\end{array}$ & $\begin{array}{l}\text { Esposti } \\
\text { et al. }{ }^{17} \\
(2015)\end{array}$ & Saúde Soc. & $\begin{array}{c}\text { Analisar o acesso ao cuidado } \\
\text { pré-natal a partir das representa- } \\
\text { ções sociais de usuárias do Sistema } \\
\text { Único de Saúde. }\end{array}$ & $\begin{array}{l}\text { Os dados permitiram compreender que o acesso ao } \\
\text { pré-natal é adequado em muitas situações, mas que } \\
\text { muitas barreiras são enfrentadas pelas gestantes para o } \\
\text { acesso a esses serviços. }\end{array}$ \\
\hline $\begin{array}{l}\text { Ouvidoria ativa em } \\
\text { saúde: avaliação } \\
\text { da qualidade da } \\
\text { atenção ao parto e } \\
\text { nascimento }\end{array}$ & $\begin{array}{l}\text { Almeida } \\
\text { et al. }{ }^{18} \\
\text { (2018) }\end{array}$ & $\begin{array}{l}\text { Rev. Saúde } \\
\text { Pública. }\end{array}$ & $\begin{array}{l}\text { Avaliar a ouvidoria ativa em saúde } \\
\text { como ferramenta de avaliação da } \\
\text { qualidade da atenção ao parto e } \\
\text { nascimento da Rede Cegonha do } \\
\text { Distrito Federal. }\end{array}$ & $\begin{array}{l}\text { Os resultados desta pesquisa podem subsidiar ações } \\
\text { para o aprimoramento da qualidade dos serviços na } \\
\text { medida em que apontam os principais aspectos da } \\
\text { Rede Cegonha que não estão adequados na região. }\end{array}$ \\
\hline
\end{tabular}




\begin{tabular}{|l|c|c|c|c|}
\hline $\begin{array}{l}\text { Avaliação da } \\
\text { assistência pré-natal } \\
\text { na perspectiva da } \\
\text { integralidade }\end{array}$ & $\begin{array}{c}\text { Costa } \\
\text { et al.19 } \\
\text { (2016) }\end{array}$ & $\begin{array}{c}\text { Rev. pesqui. } \\
\text { cuid. fundam. }\end{array}$ & $\begin{array}{c}\text { Avaliar a assistência pré-natal na } \\
\text { perspectiva da integralidade. }\end{array}$ & $\begin{array}{c}\text { Infere-se para este município, no que tange a assistên- } \\
\text { cia pré-natal, que são necessárias intervenções nesta } \\
\text { área; para alcançar um padrão de qualidade pautado } \\
\text { na integralidade. }\end{array}$ \\
\hline
\end{tabular}

\section{DISCUSSÃO}

Houve predominância de pesquisas descritivas, com variação da abordagem qualiquantitativa. Dos artigos selecionados, onze tiveram em suas conclusões a necessidade de mudanças no acompanhamento pré-natal, como o aumento da cobertura e captação precoce das gestantes e educação permanente dos profissionais de saúde, Dentre essas pesquisas, duas possuem foco principal nas gestantes negras, pardas e indígenas. Seus estudos demonstraram que após décadas ainda há muita desigualdade étnico-racial e sociodemográfica no Brasil. Um dos artigos relata o pedido de melhoria na qualidade da assistência ofertada dos Distritos Sanitários Especiais Indígenas. Expondo a urgência de atitudes à gestão e aos profissionais de saúde.

Positivamente, os três estudos que tiveram bons resultados na redução da desigualdade destacaram o enfermeiro como um importante meio de comunicação e esclarecimento de dúvidas para as gestantes, o apoio de uma equipe multidisciplinar para uma assistência continuada e a adequação às normas do PHPN e da Rede Cegonha.

Após análise sistemática dos artigos, fez-se necessário a inclusão de dois eixos temáticos: Integralidade do Cuidado Pré-natal e Qualidade da Assistência à Saúde da mulher na Atenção Básica.
Integralidade do cuidado pré-natal

Nas diretrizes do Sistema Único de Saúde encontram-se como um dos princípios a integralidade, ou seja, integração de uma rede de serviços que tem por objetivo a resolutividade de problemas da população através do livre acesso ${ }^{19,24,25,26,28}$.

Várias estratégias vêm sendo implantadas a fim de manter uma adequada atenção pré-natal às mulheres, uma vez que existe relação entre uma assistência de qualidade e índices reduzidos de morte materna e fetal. Os profissionais estão diretamente ligados a esses dados, já que suas atitudes podem meIhorar efetivamente a realidade não só das gestantes, mas de toda a família' 19,20,23,24,28.

Qualidade da assistência à saúde da muIher na atenção básica

Espera-se que os profissionais da atenção primária façam busca ativa na comunidade para captação precoce das gestantes, bem como realizar ações educativas, solicitar os exames do $1^{\circ}$ trimestre de gestação, acompanhá-la durante todo o período gestacional e realizar a consulta puerperal, tudo isso de uma maneira humanizada $21,23,24,25,26,28,29$.

Por sua vez, a Rede Cegonha amplia o acesso às unidades de saúde, assegurando os direitos à mulher, ao recém-nascido e à criança, com acolhimento de qualidade durante o pré-natal, garantindo transporte de urgência e emergência, promovendo o vínculo da gestante com a instituição de referência para realização do parto e apoiando a presença do acompanhante durante todo o parto, sendo este de escolha da gestante ${ }^{19,20,23,25,29,30,31,32}$.

\section{CONCLUSÃO}

Inicialmente, faz-se necessário salientar que uma boa assistência ao pré-natal garante a saúde do binômio mãe-filho e, para isso, deve-se buscar identificar os riscos durante o período gravídico. De acordo com os dados apresentados nesta revisão, a assistência pré-natal oferecida em alguns locais do Brasil foi classificada como ruim ou regular, levando em consideração que na maioria dos estudos foram encontrados itens a serem aprimorados.

Oportuno se torna também mencionar que a pesquisa contribuiu para avaliar a assistência prestada às gestantes, bem como as ações de acompanhamento dos profissionais de saúde durante o pré-natal na atenção básica de saúde. O que possibilitou a visualização das dificuldades relacionadas à gestão, à equipe multiprofissional, à capacitação dos profissionais de saúde e à melhoria da qualidade das consultas de pré-natal.

Portanto, resta admitir que faz-se necessário a construção de pesquisas periódicas sobre o acompanhamento e a adequação da assistência ao pré-natal dentro da atenção básica à saúde, para assim, supervisionar e controlar sistematicamente o cuidado pré-natal no Brasil ao longo dos anos.

\section{Referências}

1. Brasil. Secretaria de Atenção à Saúde. Política nacional de atenção integral à saúde da mulher: princípios e diretrizes [livro eletrônico]. Brasília: Editora do Ministério da Saúde; 2004. Disponível em: <http:// bvsms.saude.gov.br/bvs/publicacoes/politica_nac_atencao_mulher. pdf $>$.
2. Grangeiro GR, Diogenes MAR, Moura ERF. Atenção Pré-Natal no Município de Quixadá-CE segundo indicadores de processo do SISPRENATAL. Rev Esc Enferm USP. 2008;42(1).

3. Brasil. Secretaria de Estado da Saúde do Rio Grande do Sul. Departamento de Ações em Saúde. Departamento de Assistência Hospitalar e 


\section{Referências}

Ambulatorial. Guia do Pré-natal na Atenção Básica. Assessoria Técnica de Planejamento. 2018.

4. Brasil. Assistência Pré-natal: Manual técnico/equipe de elaboração: Janine Schirmer et al. - $3^{a}$ edição - Brasília: Secretaria de Políticas de Saúde - SPS/Ministério da Saúde, 2000.66p.

5. Ribeiro DC. Proposta para melhorias no Programa de Pré-Natal e Puerpério da Unidade Básica de Saúde Laurença Abreu da Silva, Baixa Grande, Município de União-Piauí. 2016. Disponível em: <https://ares. unasus.gov.br/acervo/handle/ARES/8069>.

6. Pitanguy JO. Movimento nacional e internacional de saúde e direitos reprodutivos. In: Giffin K, Costa SH, organizadores. Questões da saúde reprodutiva. Rio de Janeiro: Editora Fiocruz; 1999. p. 19-38.

7. World Health Organization. Making pregnancy safer - a health sector strategy for reducing maternal and e perinatal morbidity and mortality. Geneva: World Health Organization; 2000.

8. Abouzahr C, Wardlaw T. La mortalidad materna al término de una década: hay indicios de progreso? Bull World Health Organ 2001; 79:561-8.

9. Osis MJD. PAISM: um marco na abordagem da saúde reprodutiva no Brasil. Cad Saúde Pública 1998; 14 Suppl:25-32.

10. Costa AM. Desenvolvimento e implantação do PAISM no Brasil, In: Giffin K, Costa SH, organizadores. Questões da saúde reprodutiva. Rio de Janeiro: Editora Fiocruz; 1999. p. 319-35.

11. Brasil. Portaria n. 1.459, de 24 de junho de 2011. Institui no âmbito do Sistema Único de Saúde - SUS - a Rede Cegonha. Diário Oficial da União de junho de 2006. Disponivel em: http://bvsms.saude.gov.br/bvs/ saudelegis/gm/2011/prt1459_24_06_2011.htmL.

12. Dias JMG, Oliveira APS, Cipolotti R, Monteiro BKSM. Mortalidade materna. Revista Médica de Minas Gerais. 2015. 25(2): 173-179. Disponível em: http://rmmg.org/exportar-pdf/1771/v25n2a06.pdf.

13. BRASIL. Ministério da Saúde. Secretaria de atenção à Saúde. Departamento de atenção básica . Atenção ao pré-natal de baixo risco. Brasília: Ministério da Saúde, 2012. 316p.

14. Ferraz L, Bordignon M. Mortalidade materna no Brasil: uma realidade que precisa melhorar. Rev Baiana Saúde Pública [Internet]. 2012 [cited 2017 Apr 05];36(2):527-38. Available from: http://files.bvs.br/ upload/S/0100-0233/2012/v36n2/a3253.pdf

15. Faria DR, Sousa RC, Costa TJNM, Leite ICG. Mortalidade materna em cidade-polo de assistência na região Sudeste: tendência temporal e determinantes sociais. Rev Méd Minas Gerais [Internet]. 2012 [cited 2017 Apr 20];22(1):1-128. Available from: http:// www.rmmg.org/artigo/detalhes/121

16. Brasil. Ministério da Saúde. Manual dos Comitês de Mortalidade Materna. 3a ed. Brasília: Ministério da Saúde [Internet]. 2009 [cited 2017 Feb 17]. Available from: http://bvsms.saude.gov.br/bvs/publicacoes/manual comites mortalidade materna.pdf

17. Moreira TMM, Viana DS, Queiroz MVO, Jorge MSB. Conflitos vivenciados pelas adolescentes com a descoberta da gravidez. Rev Esc Enferm USP. 2008;42(2):312-20.

18. Mundo, Transformando Nosso. A agenda 2030 para o Desenvolvimento Sustentável.15. 2016. Disponível em: http://www.mds.gov.br/ webarquivos/publicacao/Brasil_Amigo_Pesso_Idosa/Agenda2030.pdf. 19. Silva LA, Alves VH, Rodrigues DP, Padoin SMM, Branco MBLR, Souza RMP. A qualidade de uma rede integrada: acessibilidade e cobertura no pré-natal. Revista de Pesquisa: Cuidado é Fundamental. 2015. abr-jun. 7(2):2298-2309. Disponível em: http://www.seer.unirio.br/index.php/ cuidadofundamental/article/view/3744/pdf 1537.

20. Leal NJ, Barreiro MSC, Mendes RB, Freitas CKAC. Assistência ao pré-natal: depoimento de enfermeiras. Revista de Pesquisa: Cuidado é Fundamental. 2018. jan-mar. 10(1): 113-122. Disponível em: http:// www.seer.unirio.br/index.php/cuidadofundamental/article/view/5991/ pdf_1.

21. Santiago CMC, Sousa CNS, Nóbrega LLR, Sales LKO, Morais FRR. Assistência ao pré-natal e as práticas desenvolvidas pela equipe de saúde: revisão integrativa. Revista de Pesquisa: Cuidado é Fundamental. 2017. jan-mar; 9(1): 279-288. Disponível em: http://www.seer. unirio.br/index.php/cuidadofundamental/article/view/4184/pdf_1.

22. Ferreira TLS, Melo FLACG, Araújo DV, Melo KDF, Andrade FB. Avaliação da assistência com foco na consulta de Atendimento pré-natal. Revista Ciência Plural (Online). 2017. 3(2): 4-15. Disponível em: https://periodicos.ufrn.br/rcp/article/view/12333/8986.

23. Maia VKV, Lima EFA, Leite FMC, Sousa Al, Primo CC. Avaliação dos indicadores de processo do Programa de Humanização no Pré-Natal e Nascimento e da Rede Cegonha.Revista de Pesquisa: Cuidado é Fundamental. 2017. out-dez; 9(4):1055-1060, 2017. Disponível em: http:// www.seer.unirio.br/index.php/cuidadofundamental/article/view/5794/ pdf_1.

24. Segatto MJ, Lima SBS, Kessler M, Eberhardt TD, Soares RSA, Silveira LBTD. Avaliação da assistência pré-natal em município do Sul do Brasil. Revista de Enfermagemda UFPI. 2015. abr-jun;4(2):4-10. Disponível em: https://revistas.ufpi.br/index.php/reufpi/article/view/3535/pdf.

25. Cunha AC, Lacerda JT, Alcauza MTR, Natal S.Avaliação da atenção ao pré-natal na Atenção Básica no Brasil. Revista Brasileira de Saúde Materno Infantil. 2019. abr/jun; 19(2): 459-470. Disponivel em: http:// www.scielo.br/pdf/rbsmi/v19n2/pt_1519-3829-rbsmi-19-02-0447.pdf. 26. Garnelo L, Horta BL, Escobar AL, Santos RV, Cardoso AM, Welch JR, et. al. Avaliação da atenção pré-natal ofertada às mulheres indígenas no Brasil: achados do Primeiro Inquérito Nacional de Saúde e Nutrição dos Povos indígenas. Cadernos de Saúde Pública. 2019. 35 Sup 3: e00181318. Disponível em: http://www.scielo.br/pdf/csp/v35s3/16784464-csp-35-s3-e00181318.pdf.

27. Diniz CSG, Batista LE, Kalckmann S, Schlithz AOC, Queiroz MR, Carvalho PCA. Desigualdades sociodemográficas e na assistência à maternidade entre puérperas no Sudeste do Brasil segundo cor da pele: dados do inquérito nacional Nascer no Brasil (2011-2012). Saúde e Sociedade. 2016. 25(3): 561-572. Disponível em: http://www.scielo.br/ pdf/sausoc/v25n3/1984-0470-sausoc-25-03-00561.pdf.

28. Oliveira RLA, Ferrari AP, Parada CMGL. Processo e resultado do cuidado pré-natal segundo os modelos de atenção primária: estudo de coorte. Revista Latina-Americana de Enfermagem. 2019. 27: e3058. Disponível em: http://www.scielo.br/pdf/rlae/v27/1518-8345-rlae27-e3058.pdf.

29. Valdes INF, Santos EO, Prado EV. Programa mais médicos: qualificação da atenção ao pré-natal e puerpério no âmbito da estratégia de saúde da família. Revista de APS - Atenção Primária à Saúde. 2017. jul-set; 20(3): 403 - 413. Disponível em: https://periodicos.ufjf.br/index. php/aps/article/view/16000.

30. Esposti CDD, Oliveira AE, Neto ETS, Travassos C. Representações sociais sobre o acesso e o cuidado pré-natal no Sistema Único de Saúde da Região Metropolitana da Grande Vitória, Espírito Santo. Saúde e Sociedade. 2015. 24(3): 765-779. Disponível em: http://www.scielo.br/ $\mathrm{pdf} / \mathrm{sausoc} / \mathrm{v24n3/0104-1290-sausoc-24-03-00765.pdf.}$

31. Almeida KJQ, Roure FN, Bittencourt RJ, Santos RMDB, Bittencourt FV, Gottems LBD, et. al. Ouvidoria ativa em saúde: avaliação da qualidade da atenção ao parto e nascimento. Revista de Saúde Pública. 2018. 52:76. Disponível em: http://www.scielo.br/scielo.php?script=sci_arttext\&nrm=iso\&lng=pt\&tlng=pt\&pid=S0034-89102018000100268.

32. Costa, FJLS, Camara JT, Costa KR, Serejo ECS, Pedrosa AO, Lima AKA. Avaliação da assistência pré-natal na perspectiva da integralidade. Revista de Pesquisa: Cuidado é Fundamental. 2016. abr-jun. 8(2):4563-4586. Disponível em: http://www.seer.unirio.br/index.php/ cuidadofundamental/article/view/5034/pdf_1918. 